\title{
"We're Taking Something So Human and Trying to Digitize": Provider Recommendations for mHealth in Palliative Care
}

\author{
Jennifer Dickman Portz, MSW, PhD, ${ }^{1,2}$ Kelsey Ford, MPH, ${ }^{2}$ David B. Bekelman, MD, MPH, ${ }^{1,3}$ \\ Rebecca S. Boxer, MD, Jean S. Kutner, MD, MSPH, ${ }^{1}$ Sara Czaja, PhD, \\ Kira Elsbernd, $\mathrm{MPH}^{2}$ and Sheana Bull, $\mathrm{PhD}^{2}$
}

\begin{abstract}
Background: Mobile health (mHealth) is a promising tool for improving health outcomes. However, the benefits of using mHealth in palliative care are under studied.

Objective: As a first step to designing meaningful palliative care-specific mobile applications, this research explored provider perspectives regarding the utility of mHealth in palliative care.

Design: A qualitative phenomenological study with semistructured interviews.

Setting/Subjects: Providers from multiple disciplines working in palliative care settings at an academic medical center.

Results: Thematic analysis resulted in five provider recommendations regarding the utility and design of palliative care-specific mHealth, including (i) thoughtfulness to language, context, and delivery when assessing palliative care needs; (ii) include tools for prognosis and advance care planning; (iii) tailor health and qualityof-life goals; (iv) emphasize supports for family and caregivers; and (v) consider technology abilities of older adults.

Conclusions: Palliative care providers are enthusiastic about the use of mHealth to improve care coordination, facilitate communication, enhance symptom monitoring, and improve patient-family support. However, providers have reservations about mobile functionality and depersonalized assessment and care. Providers stress the utility of mHealth to facilitate palliative care rather than replace important multidisciplinary services.
\end{abstract}

Keywords: digital health; family caregiving; health information technology; mobile device; patient reported outcomes; social support

\section{Background}

M obile health (mHealth), the use of mobile devices to facilitate health services or public health, ${ }^{1}$ is growing in popularity in health systems and among providers and patients. ${ }^{2}$ One specific type of mHealth is the use of native and hybrid mobile applications (apps), which are software programs downloaded from an app store (iOS App Store, Google Play, and so on) directly onto a mobile device, such as a smart phone or tablet, and function with and without access to the internet. Currently, there are over 318,000 commercially available hybrid and native health-related mobile apps. ${ }^{3}$ Such apps are used across a variety of health concerns and needs including chronic disease management, ${ }^{4}$ physical activity, healthy eating promotion, ${ }^{5}$ smoking cessation, ${ }^{6}$ and clinical communication. ${ }^{7}$

Palliative care-specific mobile apps, which are relatively new to the field, ${ }^{8}$ have targeted supports for cancer patients, ${ }^{9}$ prompts for provider-based palliative care consultation, and resource sharing. ${ }^{10-12}$ However, there are few palliative care apps that target both the patient and family caregivers. ${ }^{13,14}$ Often, palliative care patients are excluded from diseasespecific mHealth trials due to their advanced illness. ${ }^{15,16}$ While there are a number of positive developments in mHealth that

${ }^{1}$ Division of General Internal Medicine, University of Colorado School of Medicine Anschutz Medical Campus, Aurora, Colorado.

${ }^{2}$ mHealth Impact Lab, Colorado School of Public Health, University of Colorado Anschutz Medical Campus, Aurora, Colorado.

${ }^{3}$ Department of Medicine, Department of Veterans Affairs, Eastern Colorado Health Care System, Aurora, Colorado.

${ }^{4}$ Insitute for Health Research, Kaiser Permanente Colorado, Aurora, Colorado.

${ }^{5}$ Division of Geriatrics and Palliative Medicine, Weill Cornell Medicine, New York, New York.

Accepted August 8,2019 . 
overlap with palliative care, ${ }^{17}$ there remains opportunity to facilitate the development of apps to be deployed by palliative care teams that impact the bio-psychosocio-spiritual domains of palliative care and are inclusive of both patient and family. ${ }^{18}$

As a first step to designing meaningful palliative carespecific mobile applications, this research explored provider perspectives regarding the utility of mHealth in palliative care. This qualitative study explores provider suggestions and considerations when designing mobile apps for seriously ill populations. The objective of this study is to identify initial recommendations for tools, resources, and functionality necessary to create useful palliative care mobile apps for patients and families.

\section{Methods}

Adhering to the Consolidated Criteria for Reporting Qualitative Research, ${ }^{19}$ this exploratory qualitative study used semistructured interviews to investigate mHealth for palliative care. A phenomenological methodology ${ }^{20,21}$ was used to describe provider experiences and considerations to inform future mobile app design for palliative care patients and their family caregivers. The research team considered their epistemological ${ }^{22}$ position and bracketed ${ }^{23}$ biases to remain reflexive ${ }^{24}$ throughout the study. All study procedures were approved by the Colorado Multiple Institutional Review Board.

\section{Sample}

Participants were recruited using purposeful sampling ${ }^{25}$ from an academic medical center. Initially, the research team targeted 10 providers from a variety of disciplines working with seriously ill patients to ensure interdisciplinary feedback and expertise with seriously ill populations. Snowball sampling methods were then used, asking interview participants to suggest other possible participants, to identify relevant providers who could speak to palliative care mHealth approaches. The research team contacted all potential participants via email to inform them of the study and invite them for an interview. Responses to the recruitment email were considered informed consent, and research team email replies included information about scheduling an interview. Of the 25 providers contacted for an interview, 22 agreed to participate, and 20 interviews were completed. Of those who did not participate, one was on medical leave, two did not respond to the recruitment email, and two initially agreed to participate, but were too busy to complete the interview.

\section{Data collection}

During September 2018-February 2019, the research team conducted 20 provider interviews. The one-on-one interviews were conducted by postgraduate-trained researchers with previous qualitative experience (J.D.P. and K.F.). The interviews were held at a private location convenient to the provider, for example, a hospital meeting room or nearby offices. Interviews were $\sim 30$ to 60 -minutes in duration and audio recorded.

The research team used a semistructured interview guide to focus the interview. First, providers were asked to give general information regarding their discipline, training, and current clinical work with palliative care or seriously ill patients. Using wireframes, a set of images presented in PowerPoint that displays functional and design elements of the mobile app, participants were asked about their opinions regarding possible app functionality and suggestions for new functions. Eighteen questions and 14 PowerPoint slides with wireframe images probed providers regarding biopsychosocial and spiritual assessment issues, in-app features, potential resources, and related importance to clinical care. Examples of potential log-in, assessment, and resource screens are seen in Figure 1. The interviewer displayed an example wireframe screen and asked a specific question

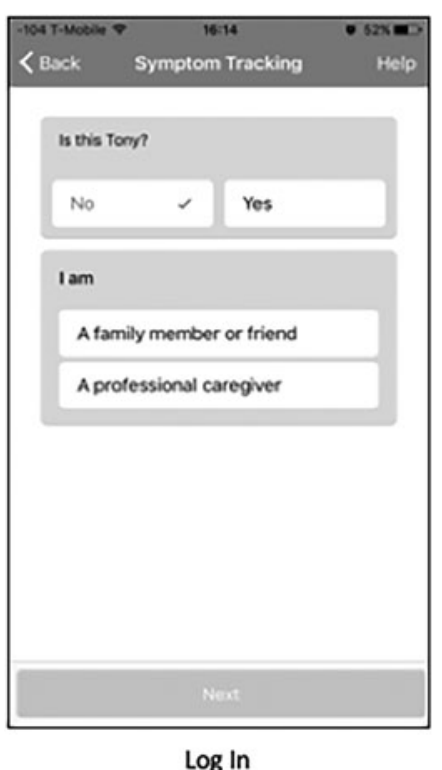

$\log \ln$
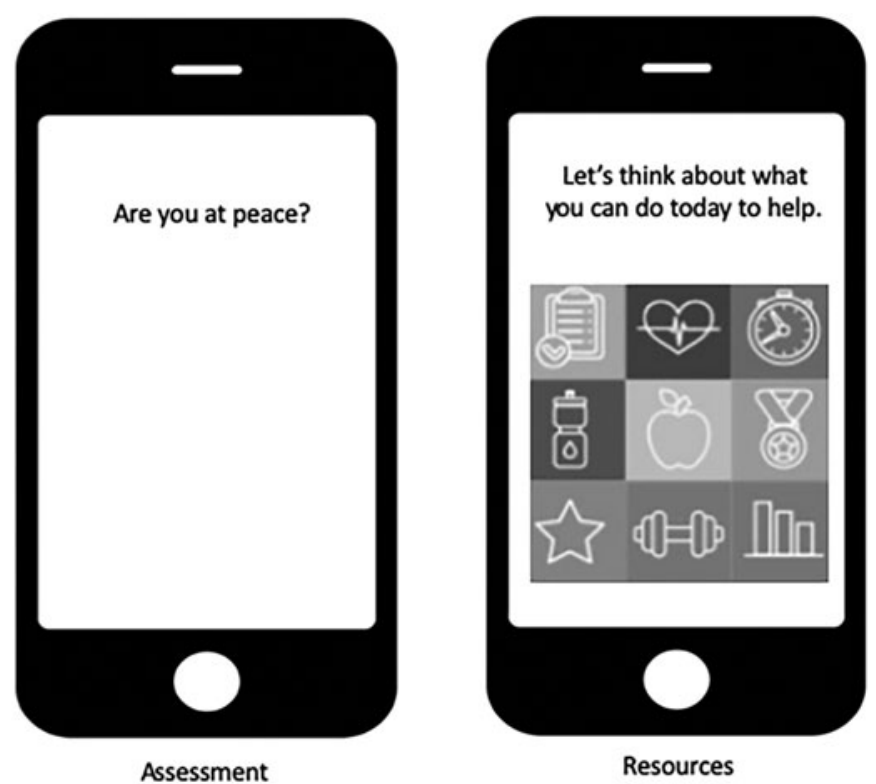

FIG. 1. Example of interview wireframes. 
such as: "do you think this would be a helpful assessment question to ask via an app? If so, do you like the way it is displayed? How would you improve the display?' Both the question guide and wireframes were modified during the data collection period iterating participant feedback. Participants were asked to brainstorm about app features that would be particularly helpful to patients and their family members. At the conclusion of the interview, participants were given a $\$ 25$ gift card.

\section{Analysis}

The principle investigator (PI) (J.D.P.) and coders (K.F. and K.E.) met regularly to review data, validate findings, and compare written notes and memos. These meetings were documented within the project audit trail ${ }^{26}$ throughout the study. Interviews were transcribed verbatim and analyzed using an iterative, team-based approach. Using the mixed methods software Dedoose (v8.035), ${ }^{27}$ coders collaboratively built consensus to develop a codebook and improve interrater reliability. Interrater reliability was calculated for three randomly selected transcripts $(81 \%$ agreement, $K=0.725)$, reflecting adequate consistency in coding across coders. The analysts triangulated ${ }^{28}$ codes with resources gathered from interviews, including health education materials currently distributed to patients and their families, other mobile application resources, and website suggestions. The research team clustered the themes into categories with significant statements to describe the core essence among responses until thematic saturation ${ }^{29}$ was reached. Illustrative quotes were coded as exemplars for each theme. As a method of member checking, ${ }^{28}$ the principal investigator met with palliative care and health technology experts to present the specific resulting themes and confirm the credibility of preliminary findings.

\section{Results}

\section{Participants}

As seen in Table 1, the sample included a diverse set of providers, including physicians, nurses, spiritual providers, social workers, and therapists. Most providers were palliative care specialists, female, and white. Provider experience with seriously ill patients ranged from 2 years to 17 years $(9.78 \pm$ 3.75 years).

Table 1. Provider Characteristics

\begin{tabular}{lc}
\hline Provider type & $(\mathrm{N}=20)$ \\
\hline Advanced practice nurse & 7 \\
Art/music therapist & 2 \\
Chaplain/spiritual care & 2 \\
Physician & 4 \\
Registered nurse & 3 \\
Social worker & 2 \\
Experience, mean & $9.78 \pm 3.75$ \\
Sex & \\
$\quad$ Female & 16 \\
$\quad$ Male & 4 \\
Race/Ethnicity & \\
$\quad$ White & 20 \\
\hline
\end{tabular}

\section{Recommendations for designing palliative care $\mathbf{m H e a l t h}$}

Thematic analysis resulted in five primary provider-based recommendations for designing mHealth. These themes are summarized in Table 2 and include thoughtfulness to language, context, and delivery when assessing palliative care needs; include tools for prognosis and advance care planning; tailor health and quality-of-life (QOL) goals; emphasize supports for the family and caregivers; and consider technology abilities of older adults.

Thoughtfulness to language, context, and delivery when assessing palliative care needs. While validated tools and assessments were described as critical in clinical practice, the validated assessment questions provided as a wireframe within an app raised concern with providers. The example assessments displayed included items for physiologic symptoms, spirituality, ${ }^{30}$ social support, ${ }^{31}$ social concerns, ${ }^{32}$ and engagement with advance care planning. ${ }^{33}$ Many providers described the questions as awkward and out of context. When not embedded within a clinical encounter, such as an office visit, the providers felt the validated assessments were inappropriate. Similarly, many providers reported the social-spiritual assessment questions are sensitive in nature, and they anticipated response hesitancy.

Many providers described the language used in the validated questions unfitting for a mobile application. Upon reviewing the wireframes, providers felt the wording in the assessments were superfluous, too high level, and nonconversational. Among these considerations, suggestions to improve language, literacy, and wording remained a key consideration when assessing both clinical and psychosocial aspects of the app. There was a strong consensus among providers to dismiss the validated wording when capturing this information. Many providers recommended alternative forms of assessment beyond a standard structured in-app survey. Many providers suggested various methods to improve the modality of validated assessments. Methods of delivery varied across all providers, some suggested interactive text messages, others considered a "chatbot" style of question and answer, and a few providers mentioned these assessments should be initiated by app and then referred to an in-person provider.

A few providers highlighted concerns regarding the overall purpose of assessment question and the data they would be collecting. Many providers described that gathering both clinical and psychosocial information is only appropriate if the device can intervene or offer support based on assessment questions.

Include tools for prognosis and advanced care planning. Many providers highlighted the criticality of expectation management around prognosis and advance care planning. This included the patient's perception of where they are on the chronic illness trajectory. In terms of an app supporting advance care planning and preparing for end of life, providers, particularly nonphysician and advance practice nurse providers, acknowledged the importance of involving family caregivers. Ideas for delivering information related to symptom tracking and illness status to providers 
Table 2. Summary of Themes with Supporting Quotes

\begin{tabular}{|c|c|}
\hline Theme & Participant feedback \\
\hline $\begin{array}{l}\text { Thoughtfulness to language, } \\
\text { context, and delivery when } \\
\text { assessing palliative care } \\
\text { needs }\end{array}$ & $\begin{array}{l}\text { "I have no problem asking these questions of people (via the app)... it is more about the } \\
\text { format... people don't want to change validated questions but you're designing } \\
\text { something for old people with a really small screen." (1015-Physician) } \\
\text { "People are going to be answering these [assessment questions] all the time... the less that } \\
\text { you absolutely you need to capture, I would say, the better... Don't ask if you can't do } \\
\text { anything," (1018-Physician) } \\
\text { "This is such a place of vulnerability for so many people and trust is the key so I feel } \\
\text { challenged at having it automated... [the resources based on assessments] are so } \\
\text { individualized and contextualized. I get uncomfortable at being prescriptive about } \\
\text { anything to do in the spiritual realm because what can work for me will not work for } \\
\text { someone else." (1023-Spiritual Provider) } \\
\text { "...We're taking something that is so human-humanistic-and trying to digitize.", } \\
\text { (1009-Art/Music Therapist) }\end{array}$ \\
\hline $\begin{array}{l}\text { Include tools for prognosis } \\
\text { and advance care planning }\end{array}$ & $\begin{array}{l}\text { "That's really nice information I think for the medical team to have if we're } \\
\text { communicating well with somebody; and also just to understand what people's actual } \\
\text { expectations are. Because they might be different than what our expectations are." } \\
\text { (1012-Spiritual Provider) } \\
\text { " ...if I can step back and look over six months' period of time and I can see that they're } \\
\text { doing this slow downward trajectory, then I better be [initiating] end of life discussions } \\
\text { or making sure things are- that they're along with me in this perception." (1005- } \\
\text { Physician) } \\
\text { "...this could be like a safe way that my dad and I could actually talk about his health } \\
\text { without actually having to talk." (1006-Social Worker) }\end{array}$ \\
\hline $\begin{array}{l}\text { Tailor Health and } \\
\text { Quality-of-Life Goals }\end{array}$ & $\begin{array}{l}\text { "...you want to help them do an activity that they would succeed in, not tell them to do } \\
\text { something they can't." (1011-Advanced Practice Nurse) } \\
\text { "With palliative care some things we talk to people about are identifying their goals or } \\
\text { their hopes. And not goals of treatment in terms of like I want to get this medication or } \\
\text { whatever... [but] the reasons they would want to get stronger. You know, to be able to } \\
\text { work in the garage again; be able to spend time with my grandkids. And if there could } \\
\text { be ways to really personalize something like this with someone's goals." (1012- } \\
\text { Spiritual Provider) }\end{array}$ \\
\hline $\begin{array}{l}\text { Emphasize supports for } \\
\text { the family and caregivers }\end{array}$ & $\begin{array}{l}\text { "... [engaging family and caregivers] seems to me something that could be useful } \\
\text { because...coming to see your doctor is not going to fix all your problems. You need to } \\
\text { be engaged in your care and you probably need some help doing it. So to me, that stands } \\
\text { out as the most valuable thing I've seen." (1018-Physician) } \\
\text { "... sometimes accepting help at home is not about you, it's actually to help your kids feel } \\
\text { better. They are more worried about you than you're worried about you. And that has } \\
\text { been more effective than other ways of trying to convince people to get more help at } \\
\text { home." (1010-Registered Nurse) } \\
\text { "...there's a lot of people who don't live close to their families... And if there were those } \\
\text { popups like it looks like you dad's having a tough day today; that's a nice...like maybe } \\
\text { today's a really good day to reach out." (1011-Advanced Practice Nurse) }\end{array}$ \\
\hline $\begin{array}{l}\text { Consider technology } \\
\text { abilities of older adults }\end{array}$ & $\begin{array}{l}\text { "I have a patient right now, who's eighty-eight years old. And I think she probably has } \\
\text { some probably more significant dementia than I thought. ... she's not ready to give up } \\
\text { her independence yet. But it's a struggle because I don't know that I trust what she's } \\
\text { providing me. So I have to have her come in." (1019-Advanced Practice Nurse) }\end{array}$ \\
\hline
\end{tabular}

and family caregivers most often included graphs focusing patient responses over time.

Tailor health and QOL goals. Providers stressed the importance of offering tailored goal setting and goalmonitoring around disease management and QOL. Goal setting focused on (i) tailoring healthy behavior goals, including increased walking or diet improvements and (ii) tailoring goals focused on QOL, including spending more time with family and friends or addressing spiritual needs. When asked about the utility of biopsychosocial assessment to monitor tailored goals via an app, providers acknowledged the importance of obtaining a more holistic sense of how the patient is doing, particularly in palliative care. Several providers also mentioned the utility of an app for reminding patients of their health and QOL goals and suggesting resources available to them. However, providers raised concerns about the ability of an mHealth algorithm to provide meaningful support for sensitive assessments.

Highlight supports for the family and caregivers. Providers consistently acknowledged that when dealing with chronic illness, caregiver burden is a challenge and discussed the importance of a team-oriented, community approach in chronic illness management and palliative care. Common themes included patient self-management, 
engagement in care, and relieving caregiver burden. Several providers offered tailored, app-generated suggestions for both the patient and caregivers of how to support each other would be valuable. Many providers told personal stories around the difficulty of communication between patients and family members. When probed, providers expressed enthusiasm with the ability to engage caregivers and care partners using an app. Several providers discussed the utility of an app to promote productive communication regarding care, thus helping family members feel updated about their loved one's condition.

Consider technology abilities of older adults. Most providers expressed hesitation about an older population's ability to access and engage with technology, and several providers spoke about their nontechnically savvy patients. However, with considerations of heuristics and delivery catered to the varying needs of an aging population, many providers agreed that an app to support palliative care would be useful. Suggestions included considerations of reading, typing, and hearing and creating modifications such as speakto-text and interoperability with hearing aid capabilities. With these considerations in mind, providers expressed value in engaging the social convoy, with greater technical abilities, to improve accessibility and utility of a palliative care mobile app.

\section{Discussion}

This study reports provider perspectives and recommendations to facilitate the design of meaningful palliative carespecific mHealth. Our results indicate providers are enthusiastic about the potential of mHealth in palliative care and also identify possible barriers and concerns.

\section{Recommendations for palliative care mHealth}

Providers have specific suggestions for how to integrate more mHealth strategies into palliative care. These recommendations provide initial insight into potential next steps for palliative care mHealth.

Use mHealth approaches to facilitate social support and advance care planning. Providers emphasized the need to include tools and supports for caregivers and family members. Previous work indicates that informal caregivers in palliative care settings are receptive to using mHealth. ${ }^{34}$ This offers a rationale for better integration of family, caregivers, and social networks into mHealth program development for palliative care. Health-related mobile applications typically target one, individual user rather than a network of users to maximize benefit. ${ }^{35}$ Only a few elements of caregiver-family support functions exist via currently available mobile applications. ${ }^{36}$ Research is needed to determine best practices for designing mHealth for simultaneous, age-diverse users of patients, caregivers, family, and social networks.

Providers highlighted the utility of mHealth for building on palliative care practices, particularly for prognostication and advance care planning, in a new and shared way. There are initiatives to use health technologies, particularly patient portals, apps, and websites, for advance care planning, ${ }^{37-39}$ but these mechanisms have yet to implement mobile functionality to target patients and their social network. People are using mobile apps, such as Snapchat and Instagram, to express emotions, events, and life events with their family, friends, and social media network. ${ }^{40}$ By incorporating these types of communication functions, palliative care mHealth may provide a unique platform for facilitating difficult conversations related to disease trajectory, goals of care, and advance directive completion. Researchers previously highlighted social media implications for end-of-life communication, ${ }^{41}$ but few empirical studies have capitalized on using social media functions ${ }^{42}$ to improve health or focus on important palliative care outcomes and services. ${ }^{43}$

Use mHealth approaches to facilitate regular assessments. One of the main benefits of mHealth for palliative care identified by providers was the ability to conduct ongoing, holistic assessments. mHealth solutions are commonly used to monitor health behaviors (physical activity and eating), ${ }^{44}$ patient reported outcomes (symptom burden, functional impact, and QOL), and sensor-captured health indicators (wireless scale for weight, blood pressure cuff for hypertension). ${ }^{45}$ However, current mHealth solutions rarely integrate various types of measurements for holistic biopsychosocial and spiritual assessment nor are these solutions offered in a palliative care population. Also, when collecting this magnitude of assessment data, that is, "big data," it is important to consider who these data are for and the best use practices. Further research is needed to investigate the maximum value of mHealth patient reported data to determine if data are better-used by providers to improve care or by patients and families to improve heath engagement. ${ }^{46,47}$

\section{Special considerations}

Although there is enthusiasm for mHealth in palliative care, the study providers offer important considerations. Providers also provide specific recommendations to address caution regarding the use of mHealth among patients with serious illness and their families.

Take care to facilitate sensitive communication appropriately with mHealth tools. Providers noted concerns regarding the dehumanization of people's disease experience by using mHealth. This speaks to the ongoing discussion related to potentially negative impacts of health technology in medical care ${ }^{48,49}$ on provider-patient relationships, personalization, and work flows. Based on our results, palliative care-specific mHealth should be considered a facilitator of supports rather than a replacement of important multidisciplinary services.

These findings also illustrate a challenge of integrating assessment tools that have been validated in clinical contexts in new mHealth modalities. The provider suggestions for deemphasizing language and specific wording from validated tools acknowledge how communication via mHealth media follows a different set of expectations and cultural norms. Although providers may need validated assessments for research or medical record documentation, using exact items or scales may not be the best format for a mobile application. Despite limited evidence in this area, researchers recommend using the Brief Pain Inventory to measure cancer-specific pain via mHealth. ${ }^{50}$ More research is needed to assess the 
alternative form reliability and responsiveness of using validated palliative care assessments via mHealth platforms.

As providers note, designers must be thoughtful of the context and delivery of sensitive health information in the setting of palliative care. The use of technology in delivering difficult news or end-of-life information can be perceived negatively by patients and their family. For example, in a recent qualitative study, patients, family members, and oncologists indicated that discussing laboratories, scans, and diagnosis via a patient portal can be challenging due to the sensitive nature of the information. ${ }^{51}$

Be certain to consider user experience and to address varies experiences with technology in designing approaches. As many palliative care patients and caregivers are older adults, providers recommended careful attention be made to mobile application design for this age group. There are specific guidelines for designing technologies for older adults $^{52}$ to improve adoption and usability. User-centered design methods, that is, the inclusion of older adults in the design process, and interdisciplinary collaborative approaches are also recommended for developing mHealth for older users. ${ }^{53}$ Despite common misconceptions, older adults and caregivers are interested in using technologies for health information and health care services. ${ }^{54,55}$

\section{Future research for designing palliative care mobile applications}

While this article provides general recommendations, additional research is needed to provide structured guidance to clinicians, researchers, and app designers who are interested in developing apps specifically for palliative care. Unique challenges exist when designing for people with serious illness and the people who help them with their care. Popular mHealth engagement strategies may not be helpful or feasible. Research is needed to explore ways to soften or improve palliative care assessments that have primarily been used in clinical or in-person, specifically investigating which modalities are most accepted and useful for this population, for example, testing patient and caregiver perceptions of chat bot functions, voice interactions, and avatars to cushion sensitive questions regarding serious illness. Further investigation is needed to test digital strategies such as nudges, ecological momentary interventions, and health communication strategies (e.g., text, voice, and pop-up functions) that can promote user engagement with specific palliative care mHealth tools. Such strategies will inform design decisions for goal setting functions such as open-ended versus multiple-choice selection design features. Finally, future work is also needed to design algorithms for assessing and intervening with multiple simultaneous users that include the patient, family, and caregivers. Despite the future research needed, this work offers incremental advancements to improve the technology supporting interventions for patients with serious illness, their family, caregivers, and health care providers.

\section{Limitations}

Although this was a comprehensive qualitative investigation, there are several limitations to our study. Data were collected with providers from one academic medical center.
Providers in various geographic or more rural areas may offer differing opinions and recommendations. Although we achieved variability in interviewing providers from multiple disciplines, the sample was also limited to white participants resulting in a lack of perspectives from underrepresented clinicians. Furthermore, by using a semistructured interview with wireframes, the interviews may have been too restrictive to fully capture providers' experiences and opinions.

\section{Conclusions}

While palliative care providers are enthusiastic about the use of mHealth to improve care coordination, facilitate communication, symptom monitoring, and patient-family support, they have reservations about mobile functionality and depersonalized assessment and care. Providers stress the utility of mHealth to facilitate palliative care for patient, caregivers, and family rather than replace important multidisciplinary services. These recommendations provide direction for the design and development of palliative care mHealth that may lead to improved health outcomes for people with serious illness and their families.

\section{Funding Information}

This research is funded by career development awards from the National Institute on Aging (K76AG059934) and the American Heart Association (18CDA34110092), and training support from National Institute on Aging (T32AG044296).

\section{Author Disclosure Statement}

No competing financial interests exist.

\section{References}

1. Telemedicine and Telehealth: HealthIT.gov. https://healthit .gov/topic/health-it-initiatives/telemedicine-and-telehealth 2017. (Last accessed February 1, 2019).

2. Labrique A, Vasudevan L, Mehl G, et al.: Digital health and health systems of the future. Glob Health Sci Pract 2018;6(Suppl 1):S1-S4.

3. The Growing Value of Digital Health: IQVIA Institute for Human Data Science. https://iqvia.com/institute/reports/thegrowing-value-of-digital-health 2017. (Last accessed February 1, 2019).

4. Agnihothri S, Cui L, Delasay M, Rajan B: The value of mHealth for managing chronic conditions. Health Care Manag Sci 2018; [Epub ahead of print]; DOI: 10.1007/ s10729-018-9458-2.

5. Vlahu-Gjorgievska E, Mulakaparambil Unnikrishnan S, Win KT: mHealth applications: A tool for behaviour change in weight management. Stud Health Technol Inform 2018;252:158-163.

6. Do H, Tran B, Pham Q, et al.: Which eHealth interventions are most effective for smoking cessation? A systematic review. Patient Prefer Adherence 2018;12:2065-2084.

7. McKay FH, Cheng C, Wright A, et al.: Evaluating mobile phone applications for health behaviour change: A systematic review. J Telemed Telecare 2018;24:22-30.

8. Ostherr K, Killoran P, Shegog R, Bruera E: Death in the digital age: A systematic review of information and communication technologies in end-of-life care. J Palliat Med 2016;19:408-420. 
9. Worster B, Swartz K: Telemedicine and palliative care: An increasing role in supportive oncology. Curr Oncol Rep 2017;19:37.

10. Meghani SH, MacKenzie MA, Morgan B, et al.: Cliniciantargeted mobile apps in palliative care: A systematic review. J Palliat Med 2017;20:1139-1147.

11. Zhang H, Liu D, Marks S, et al.: Usage patterns of a mobile palliative care application. J Palliat Med 2018;21:796-801.

12. Cox CE, Jones DM, Reagan W, et al.: Palliative care planner: A pilot study to evaluate acceptability and usability of an electronic health records system-integrated, needs-targeted app platform. Ann Am Thorac Soc 2018; 15:59-68.

13. Pinto S, Caldeira S, Martins JC: e-Health in palliative care: Review of literature, Google play and App store. Int J Palliat Nurs 2017;23:394-401.

14. MacKenzie Greenle M, Morgan B, Sayani S, Meghani SH: Identifying mobile apps targeting palliative care patients and family members. J Palliat Med 2018;21:1380-1385.

15. Head BA, Schapmire TJ, Zheng Y: Telehealth in palliative care: A systematic review of patient-reported outcomes. J Hosp Palliat Nurs 2017;19:130-139.

16. Capurro D, Ganzinger M, Perez-Lu J, Knaup P: Effectiveness of eHealth interventions and information needs in palliative care: A systematic literature review. J Med Internet Res 2014;16:e72.

17. Marcolino MS, Oliveira JAQ, D’Agostino M, et al.: The impact of mHealth interventions: Systematic review of systematic reviews. JMIR MHealth UHealth 2018;6:e23.

18. Nwosu AC, Mason S: Palliative medicine and smartphones: An opportunity for innovation? BMJ Support Palliat Care 2012;2:75-77.

19. Tong A, Sainsbury P, Craig J: Consolidated criteria for reporting qualitative research (COREQ): A 32-item checklist for interviews and focus groups. Int $\mathrm{J}$ Qual Health Care 2007;19:349-357.

20. Sokolowski R: Introduction to Phenomenology. Cambridge, United Kingdom: Cambridge University Press, 2000, $238 \mathrm{p}$.

21. Starks H, Brown Trinidad S: Choose your method: A comparison of phenomenology, discourse analysis, and grounded theory. Qual Health Res 2007;17:1372-1380.

22. Carter SM, Little M: Justifying knowledge, justifying method, taking action: Epistemologies, methodologies, and methods in qualitative research. Qual Health Res 2007;17: 1316-1328.

23. Giorgi A: The Descriptive Phenomenological Method in Psychology: A Modified Husserlian Approach. Pittsburgh, PA: Duquesne University Press, 2009, 233 p.

24. Kralik D: Reflexivity: A practical guide for researchers in health and social sciences. J Adv Nurs 2005;50:227.

25. Creswell JW, Poth CN: Qualitative Inquiry and Research Design: Choosing Among Five Approaches, 4th ed. Los Angeles, CA: SAGE, 2018, 459 p.

26. Silver C, Lewins A: Using Software in Qualitative Research: A Step-by-Step Guide London, United Kingdom: SAGE Publications Ltd. http://methods.sagepub.com/book/ using-software-in-qualitative-research-2e 2014. (Last accessed February 21, 2019).

27. Dedoose: Los Angeles, CA: SocioCultural Research Consultants, LLC. www.dedoose.com 2018.

28. Mays N: Qualitative research in health care: Assessing quality in qualitative research. BMJ 2000;320:50-52.
29. Ando H, Cousins R, Young C: Achieving saturation in thematic analysis: Development and refinement of a codebook. Compr Psychol 2014;3:03.CP.3.4.

30. Steinhauser KE: "Are you at peace?": One item to probe spiritual concerns at the end of life. Arch Intern Med 2006; 166:101

31. Broadhead WE, Gehlbach SH, de Gruy FV, Kaplan BH: The Duke-UNC Functional Social Support Questionnaire. Measurement of social support in family medicine patients. Med Care 1988;26:709-723.

32. Reese DJ, Raymer M, Orloff SF, et al.: The Social Work Assessment Tool (SWAT). J Soc Work End Life Palliat Care 2006;2:65-95.

33. Sudore RL, Heyland DK, Barnes DE, et al.: Measuring advance care planning: Optimizing the advance care planning engagement survey. J Pain Symptom Manage 2017; 53:669-681.e8.

34. Phongtankuel V, Shalev A, Adelman RD, et al.: Mobile health technology is here-but are hospice informal caregivers receptive? Am J Hosp Palliat Med 2018;35:15471552.

35. Wolff JL, Darer JD, Larsen KL: Family caregivers and consumer health information technology. J Gen Intern Med 2016;31:117-121.

36. Grossman MR, Zak DK, Zelinski EM: Mobile apps for caregivers of older adults: Quantitative content analysis. JMIR MHealth UHealth 2018;6:e162.

37. Bose-Brill S, Feeney M, Prater L, et al.: Validation of a novel electronic health record patient portal advance care planning delivery system. J Med Internet Res 2018;20: e208.

38. Fine RL, Yang Z, Spivey C, et al.: Early experience with digital advance care planning and directives, a novel consumer-driven program. Proc Bayl Univ Med Cent 2016; 29:263-267.

39. Jordan SR, Brungardt A, Phimphasone-Brady P, Lum HD: Patient perspectives on advance care planning via a patient portal. Am J Hosp Palliat Med 2019;36:682-687.

40. Smith A, Anderson M: The Demographics of Social Media Users-2018. Washington, DC: Pew Research Center. https://pewinternet.org/2018/03/01/social-media-use-in-2018/ 2018. (Last accessed April 2, 2019).

41. Taubert M, Watts G, Boland J, Radbruch L: Palliative social media. BMJ Support Palliat Care 2014;4:13-18.

42. Park A, Bowling J, Shaw G, et al.: Adopting social media for improving health: Opportunities and challenges. N C Med J 2019;80:240-243.

43. Taubert M: Social media in palliative medicine research: Jump into the cool water. BMJ Support Palliat Care 2018;8: 292-293.

44. McCallum C, Rooksby J, Gray CM: Evaluating the impact of physical activity apps and wearables: Interdisciplinary review. JMIR MHealth UHealth 2018;6:e58.

45. Donevant SB, Estrada RD, Culley JM, et al.: Exploring app features with outcomes in mHealth studies involving chronic respiratory diseases, diabetes, and hypertension: A targeted exploration of the literature. J Am Med Inform Assoc 2018;25:1407-1418.

46. Tanuseputro P: Delivering care to those in need: Improving palliative care using linked data. Palliat Med 2017;31:489491.

47. Weiler A: mHealth and big data will bring meaning and value to patient-reported outcomes. mHealth 2016;2:2. 
48. Naylor CD: On the prospects for a (deep) learning health care system. JAMA 2018;320:1099.

49. Bailey J: Does health information technology dehumanize health care? Virtual Mentor 2011;13:181-185.

50. Abahussin AA, West RM, Wong DC, Ziegler LE: PROMs for pain in adult cancer patients: A systematic review of measurement properties. Pain Pract 2019;19:93-117.

51. Alpert JM, Morris BB, Thomson MD, et al.: Implications of patient portal transparency in oncology: Qualitative interview study on the experiences of patients, oncologists, and medical informaticists. JMIR Cancer 2018;4:e5.

52. Fisk AD, Rogers WA, Charness N, et al.: Designing for Older Adults: Principles and Creative Human Factors Approaches, 2nd ed. Boca Raton, FL: CRC Press, 2009, 277 p. (Human factors and aging series).

53. Matthew-Maich N, Harris L, Ploeg J, et al.: Designing, implementing, and evaluating mobile health technologies for managing chronic conditions in older adults: A scoping review. JMIR MHealth UHealth 2016;4:e29.
54. Fox S, Duggan M, Purcell K: Family Caregivers are Wired for Health. Washington, DC: Pew Research Center. http:// pewinternet.org/2013/06/20/family-caregivers-are-wiredfor-health/ 2013. (Last accessed August 15, 2017).

55. Anderson M, Perrin A: Tech Adoption Climbs Among Older Adults. Washington, DC: Pew Research Center. https://pewinternet.org/2017/05/17/technology-use-amongseniors/ 2017. (Last accessed April 3, 2019).

Address correspondence to: Jennifer Dickman Portz, MSW, PhD

Division of General Internal Medicine University of Colorado School of Medicine Anschutz. Medical Campus Mailstop B180 12631 East 17th Avenue Aurora, CO 80045

E-mail: jennifer.portz@cuanschutz.edu 